\title{
ANGLE DISTORTION THEOREMS FOR STARLIKE \\ AND SPIRALLIKE FUNCTIONS WITH RESPECT \\ TO A BOUNDARY POINT
}

MARK ELIN AND DAVID SHOIKHET

Received 21 December 2005; Revised 16 April 2006; Accepted 25 April 2006

We exhibit angle bounds for starlike and spirallike functions with respect to a boundary point. As an application, we obtain a covering theorem for functions convex in one direction.

Copyright (c) 2006 Hindawi Publishing Corporation. All rights reserved.

\section{Introduction}

Let $\Delta$ be the open unit disk in the complex plane $\mathbb{C}$. $\operatorname{By} \operatorname{Hol}(\Delta, \mathbb{C})$ we denote the set of all holomorphic functions in $\Delta$.

Definition 1.1. A univalent function $h$ on $\Delta$ is called spirallike (resp., starlike) on $\Delta$ if for some $\mu \in \mathbb{C}$ with $\operatorname{Re} \mu>0$ (resp., $\mu \in \mathbb{R}$ with $\mu>0$ ) and for each $t \geq 0$, the element $e^{-\mu t} h(z)$ belongs to $h(\Delta)$ whenever $z \in \Delta$.

It is clear that $0 \in \overline{h(\Delta)}$. Moreover,

(i) if $0 \in h(\Delta)$, then $h$ is called spirallike (resp., starlike) with respect to an interior point;

(ii) if $0 \notin h(\Delta)$, then $h$ is called spirallike (resp., starlike) with respect to a boundary point. In this case, there is a boundary point (say, $z=1$ ) such that $h(1):=$ $\angle \lim _{z \rightarrow 1} h(z)=0$ (see, e.g., $[1,6]$ ); by symbol $\angle \lim$, we denote the angular (nontangential) limit of a function at a boundary point of $\Delta$.

The class of spirallike (starlike) functions with respect to a boundary point normalized by the conditions $h(1)=0$ and $h(0)=1$ will be denoted by Spiral [1] (resp., Star [1]).

It was proved in $[1,7]$ that for any function $h \in$ Spiral [1], the limit of the so-called Visser-Ostrowski quotient

$$
\angle \lim _{z \rightarrow 1} \frac{(z-1) h^{\prime}(z)}{h(z)}=\mu
$$

exists with $\mu \in \Omega$, where $\Omega=\{\lambda:|\lambda-1| \leq 1, \lambda \neq 0\}$. 
For given $\mu \in \Omega$, we denote the class of spirallike functions which satisfy (1.1) by Spiral $_{\mu}[1]$. If the number $\mu$ is real, that is, $0<\mu \leq 2$, then the function $h$ is, in fact, starlike, see [1]. If this is the case, we write $h \in \operatorname{Star}_{\mu}[1]$.

It turns out that the number $\mu$ can be used for some geometric characteristic of the image $h(\Delta)$. Namely, it was shown in [7] that if $\mu$ is real, then the smallest wedge with vertex at the origin which contains $h(\Delta)$ is exactly of angle $\mu \pi$.

In this paper, we establish an angle distortion theorem for a class of unbounded starlike and spirallike functions which gives us both covering and growth estimates.

\section{Starlike and spirallike functions}

Definition 2.1 (see [17]). Say that a univalent function $h$ belongs to the class $\operatorname{Spiral}_{\mu, \nu}[1$, $-1]$ if it is of the class $\operatorname{Spiral}_{\mu}[1]$ and satisfies the conditions

$$
\begin{gathered}
\angle \lim _{z \rightarrow-1} h(z)=\infty \\
\angle \lim _{z \rightarrow-1} \frac{h^{\prime}(z)(z+1)}{h(z)}=v \neq 0 .
\end{gathered}
$$

It was shown in [17] that if $\mu$ is a real number (i.e., $0<\mu \leq 2$ ), then so is $\nu$, and $-\mu \leq$ $\nu<0$. Since in this case $h$ is starlike, we will write $h \in \operatorname{Star}_{\mu, \nu}[1,-1]$. First we consider this class.

Theorem 2.2. (i) If $h \in \operatorname{Star}_{\mu}[1]$, then the wedge

$$
W^{*}=\left\{w \in \mathbb{C}:\left|\arg w-\theta_{*}\right|<\frac{\mu \pi}{2}\right\},
$$

where $\theta^{*}:=\lim _{x \rightarrow 1^{-}} \arg h(x)$, is the smallest one which contains the image $h(\Delta)$.

(ii) If $h \in \operatorname{Star}_{\mu, \nu}[1,-1]$, then the wedge

$$
W_{*}=\left\{w \in \mathbb{C}:\left|\arg w-\theta_{*}\right|<\frac{|\nu| \pi}{2}\right\}
$$

where

$$
\theta_{*}:=\lim _{x \rightarrow-1^{+}} \operatorname{argh}(x)
$$

is contained in $h(\Delta)$, and there is no wedge $W \neq W_{*}$ with $W_{*} \subset W \subset h(\Delta)$.

Conversely, if $h \in \operatorname{Star}_{\mu}[1]$ and the image $h(\Delta)$ contains some open wedge, then there are $\nu(-\mu \leq \nu<0)$ and an automorphism $\Psi$ of the open unit disk $\Delta$ such that function $(1 / h(\Psi(0))) h \circ \Psi$ belongs to $\operatorname{Star}_{\mu, \nu}[1,-1]$ (see Figure 2.1).

To prove this theorem, we need an integral representation for the classes $\operatorname{Star}_{\mu}[1]$ and $\operatorname{Star}_{\mu, \nu}[1,-1]$. By $\delta(\tau)$ we denote the Dirac $\delta$-function (measure) at the point $\tau$. 


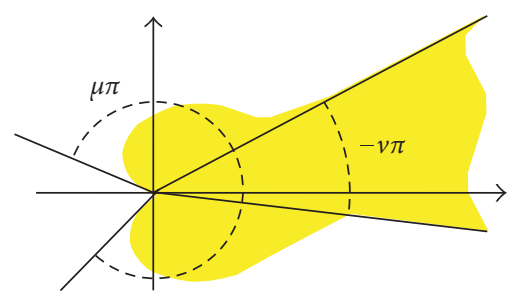

Figure 2.1. Angle distortion.

Lemma 2.3. Let $0<\mu \leq 2$. A function $h \in \operatorname{Hol}(\Delta, \mathbb{C})$ is of the class $\operatorname{Star}_{\mu}[1]$ if and only if it admits the following representation:

$$
h(z)=(1-z)^{\mu}(1+z)^{\kappa} \exp \left[-(\mu+\kappa) \oint_{\partial \Delta} \log (1-z \bar{\zeta}) d \sigma(\zeta)\right]
$$

with some probability measure $d \sigma$ on the unit circle which is mutually singular relative to both $\delta(1)$ and $\delta(-1)$ and with some $\kappa,-\mu \leq \kappa \leq 0$. Furthermore, $h \in \operatorname{Star}_{\mu, \nu}[1,-1]$ if and only if (2.5) holds with $\kappa=v<0$.

Proof. It was shown in [1] that any function $h \in$ Spiral[1] does satisfy a generalized Robertson's inequality (see [15])

$$
\operatorname{Re}\left[\frac{2 z h^{\prime}(z)}{\lambda h(z)}+\frac{1+z}{1-z}\right]>0
$$

with some $\lambda, \operatorname{Re} \lambda>0$. Moreover, $h \in \operatorname{Spiral}_{\mu}[1]$ if and only if (2.6) holds for $\lambda=R \mu, R \geq$ 1 , where $\mu$ is defined by (1.1) (see [1]).

As mentioned, if number $\lambda$ in (2.6) (hence, $\mu$ in (1.1)) is real, then $h$ is actually starlike.

So, let $h \in \operatorname{Star}_{\mu}[1], 0<\mu \leq 2$. By the Riesz-Herglotz representation, we have

$$
\frac{2 z h^{\prime}(z)}{h(z)}=\mu \oint_{\partial \Delta}\left(\frac{1+z \bar{\zeta}}{1-z \bar{\zeta}}-\frac{1+z}{1-z}\right) d \tilde{\sigma}(\zeta)
$$

with some probability measure $d \tilde{\sigma}$. Integrating this equality, we get

$$
h(z)=(1-z)^{\mu} \exp \left[-\mu \oint_{\partial \Delta} \log (1-z \bar{\zeta}) d \tilde{\sigma}(\zeta)\right] .
$$

Decompose $d \tilde{\sigma}$ relative to the Dirac measure $\delta(1)$, that is,

$$
d \tilde{\sigma}=a \delta(1)+d \sigma_{1}
$$

where $\delta(1)$ and $d \sigma_{1}$ are mutually singular positive measures and $0 \leq a \leq 1$. If $a \neq 0$, then substituting (2.9) into (2.8) and differentiating this equality, we get that inequality (2.6) holds with $\lambda=\mu(1-a)$ which is smaller than $\mu$. This contradiction shows that $a=0$, that is, the measures $d \tilde{\sigma}$ and $\delta(1)$ are mutually singular. 
Also one can write

$$
d \tilde{\sigma}=b \delta(-1)+(1-b) d \sigma, \quad 0 \leq b \leq 1,
$$

where the probability measures $d \sigma$ and $\delta(-1)$ are mutually singular. Using this decomposition, we rewrite (2.8) as follows:

$$
h(z)=(1-z)^{\mu}(1+z)^{-b \mu} \exp \left[-(1-b) \mu \oint_{\partial \Delta} \log (1-z \bar{\zeta}) d \sigma(\zeta)\right] .
$$

This formula coincides with (2.5) for $\kappa=-b \mu$.

To continue, we evaluate the limit of the Visser-Ostrowski quotient at the point $z=-1$ :

$$
\begin{aligned}
\angle \lim _{z \rightarrow-1} \frac{h^{\prime}(z)(z+1)}{h(z)} & =\angle \lim _{z \rightarrow-1}(z+1)\left[\frac{-\mu}{1-z}-\frac{b \mu}{1+z}-(1-b) \mu \oint_{\partial \Delta} \frac{\bar{\zeta}}{1-z \bar{\zeta}} d \sigma(\zeta)\right] \\
& =-b \mu-(1-b) \mu \angle \lim _{z \rightarrow-1} \oint_{\partial \Delta} \frac{\bar{\zeta}(1+z)}{1-z \bar{\zeta}} d \sigma(\zeta) .
\end{aligned}
$$

Since

$$
\left|\frac{\bar{\zeta}(1+z)}{1-z \bar{\zeta}}\right| \leq \frac{|1+z|}{1-|z|},
$$

we see that the integrand in the last expression of (2.12) is bounded on each nontangential approach region $\Gamma_{\alpha}=\{w:|1+z| /(1-|z|)<\alpha\}, \alpha>1$, at the point $z=-1$. Since the measures $d \sigma$ and $\delta(-1)$ are mutually singular, we conclude by Lebesgue's bounded convergence theorem that the last integral in (2.12) is equal to zero, so

$$
\angle \lim _{z \rightarrow-1} \frac{h^{\prime}(z)(z+1)}{h(z)}=-b \mu(=\kappa) .
$$

Hence $h \in \operatorname{Star}_{\mu, \nu}[1,-1]$ if and only if $b \neq 0$ and then $\nu=\kappa$.

Proof of Theorem 2.2. Assertion (i) was proved in [9]. Now we prove assertion (ii).

First we show that the image $h(\Delta)$ contains the wedge $W_{*}$ defined by (2.3). Since $\angle \lim _{z \rightarrow-1} h(z)=\infty$, for each $\delta \in(0, \pi / 2)$ and each $R>0$, there exists $r_{0}>0$ such that for all $r \in\left(0, r_{0}\right)$,

$$
|h(z)|>R
$$

whenever

$$
z \in D_{r, \delta}:=\{z \in \Delta:|1+z| \leq r,|\arg (1+z)| \leq \delta\} .
$$

Setting $\kappa=v$ in (2.5), we obtain by Lebesgue's bounded convergence theorem that the following limit exists:

$$
\lim _{z \rightarrow-1} \arg \frac{h(z)}{(1+z)^{\nu}}=-(\mu+\nu) \lim _{z \rightarrow-1} \oint_{\partial \Delta} \arg (1-z \bar{\zeta}) d \sigma(\zeta) .
$$


On the other hand, by formula (2.5), we have

$$
\theta_{*}=\lim _{x \rightarrow-1^{+}} \arg h(x)=-(\mu+\nu) \lim _{x \rightarrow-1} \oint_{\partial \Delta} \arg (1-x \bar{\zeta}) d \sigma(\zeta) .
$$

Therefore,

$$
\lim _{z \rightarrow-1} \arg \frac{h(z)}{(1+z)^{\nu}}=\theta_{*}
$$

Thus, decreasing $r$ (if it is necessary), we have

$$
\theta_{*}-\varepsilon<\arg \frac{h(z)}{(1+z)^{v}}<\theta_{*}+\varepsilon
$$

for all $z \in D_{r, \delta}$. So, if $z$ belongs to the arc

$$
\Gamma:=\{z \in \Delta:|1+z|=r,|\arg (1+z)| \leq \delta\} \subset D_{r, \delta},
$$

that is, $z=-1+r e^{i t},|t| \leq \delta$, then

$$
\theta_{*}-\varepsilon-t|\nu|<\operatorname{argh}(z)<\theta_{*}+\varepsilon-t|\nu| .
$$

In particular,

$$
\begin{gathered}
\operatorname{argh}\left(-1+r e^{i \delta}\right)<\theta_{*}+\varepsilon-\delta|\nu|, \\
\operatorname{argh}\left(-1+r e^{-i \delta}\right)>\theta_{*}-\varepsilon+\delta|\nu| .
\end{gathered}
$$

Therefore the curve $h(\Gamma)$ lies outside the disk $|z| \leq R$ and joins two points having arguments less than $\theta_{*}+\varepsilon-\delta|\nu|$ and greater than $\theta_{*}-\varepsilon+\delta|\nu|$, respectively. Since $h$ is starlike, we see that $h(\Delta)$ contains the sector

$$
\left\{w \in \mathbb{C}:|w|<R,\left|\arg w-\theta_{*}\right|<\delta|\nu|-\varepsilon\right\} .
$$

As $R$ and $\varepsilon$ are arbitrary, one concludes that

$$
\left\{w \in \mathbb{C}:\left|\arg w-\theta_{*}\right|<\delta|\nu|\right\} \subset h(\Delta) .
$$

Letting $\delta \rightarrow \pi / 2$, we obtain that $W_{*} \subset h(\Delta)$.

Now, since $h$ is a starlike function, $\arg h\left(e^{i \varphi}\right)$ is an increasing function in $\varphi \in(0,2 \pi)$. So the limits

$$
\lim _{\varphi \rightarrow \pi^{ \pm}} \operatorname{argh}\left(e^{i \varphi}\right)
$$

exist. In addition, one can find two sequences $\varphi_{n,+} \rightarrow \pi^{+}$and $\varphi_{n,-} \rightarrow \pi^{-}$such that the values $h\left(e^{i \varphi_{n, \pm}}\right)$ are finite. Once again, by Lemma 2.3, we get

$$
\lim _{n \rightarrow \infty} \arg h\left(e^{i \varphi_{n,+}}\right)-\arg h\left(e^{i \varphi_{n,-}}\right)=\lim _{n \rightarrow \infty} \nu\left(\arg \left(1+e^{i \varphi_{n,+}}\right)-\arg \left(1+e^{i \varphi_{n,-}}\right)\right)=|\nu| \pi .
$$


Therefore the image contains no wedge $W$ of angle larger than $|\nu| \pi$ with $W_{*} \subset W \subset$ $h(\Delta)$.

To prove the converse assertion, suppose that the open wedge

$$
W=\{w \in \mathbb{C}:|\arg w-\alpha|<\beta\}
$$

lies in $h(\Delta)$. Then the curve $\ell:=h^{-1}(\{w: \arg w=\alpha\})$ lies in $\Delta$ and joins the point 1 with another boundary point $\eta$. Denote by $\Psi$ an automorphism of $\Delta$ such that $\Psi(1)=1$ and $\Psi(-1)=\eta$. Define $h_{1} \in \operatorname{Hol}(\Delta, \mathbb{C})$ by $h_{1}=(1 / h(\Psi(0))) h \circ \Psi$. It is clear that $h_{1}(\Delta)=$ $(1 / h(\Psi(0))) h(\Delta)$. Consequently, $h_{1}$ is a starlike function with respect to a boundary point, and $W_{1} \subset h_{1}(\Delta)$, where

$$
W_{1}=\left\{w \in \mathbb{C}:\left|\arg w-\alpha_{1}\right|<\beta\right\}, \quad \alpha_{1}=\alpha-\operatorname{argh}(\Psi(0)) .
$$

A simple calculation shows that $h_{1} \in \operatorname{Star}_{\mu}[1]$ and $\angle \lim _{z \rightarrow-1} h_{1}(z)=\infty$. We now show that $h_{1} \in \operatorname{Star}_{\mu, \nu}[1,-1]$ for $\nu=-2 \beta / \pi<0$.

Since $W_{1}$ cannot be extended to a larger wedge lying in $h_{1}(\Delta)$, for each $\varepsilon>0$ there are boundary points of the image $h_{1}(\Delta)$ belonging to

$$
\begin{aligned}
& \left\{w: \alpha_{1}-\beta-\varepsilon<\arg w \leq \alpha_{1}-\beta\right\}, \\
& \left\{w: \alpha_{1}+\beta \leq \arg w \leq \alpha_{1}+\beta+\varepsilon\right\} .
\end{aligned}
$$

Let $\left\{\phi_{n}^{+}\right\}$be a decreasing sequence such that $\phi_{n}^{+} \rightarrow \arg \eta$, the values $h_{1}\left(e^{i \phi_{n}^{+}}\right)$exist, and $\alpha_{1}+\beta \leq \arg h_{1}\left(e^{i \phi_{n}^{+}}\right)<\alpha_{1}+\beta+1 / n$. Similarly, let $\left\{\phi_{n}^{-}\right\}$be an increasing sequence such that $\phi_{n}^{-} \rightarrow \arg \eta$, the values $h_{1}\left(e^{i \phi_{n}^{-}}\right)$exist, and $\alpha_{1}-\beta-1 / n<\arg h_{1}\left(e^{i \phi_{n}^{-}}\right) \leq \alpha_{1}-\beta$.

By Lemma 2.3, we have

$$
\arg h_{1}(z)=\mu \arg (1-z)+\kappa \arg (1+z)-(\mu+\kappa) \oint_{\partial \Delta} \arg (1-z \bar{\zeta}) d \sigma(\zeta)
$$

where the measure $d \sigma$ is singular relative to $\delta(-1)$ and $-\mu \leq \kappa \leq 0$.

Consider the expression $\arg h_{1}\left(e^{i \phi_{n}^{+}}\right)-\arg h_{1}\left(i e^{\phi_{n}^{-}}\right)$which tends to $2 \beta$ :

$$
\begin{aligned}
& \arg h_{1}\left(e^{i \phi_{n}^{+}}\right)-\arg h_{1}\left(e^{i \phi_{n}^{-}}\right)= \mu\left[\arg \left(1-e^{i \phi_{n}^{+}}\right)-\arg \left(1-e^{i \phi_{n}^{-}}\right)\right] \\
&+\kappa\left[\arg \left(1+e^{i \phi_{n}^{+}}\right)-\arg \left(1+e^{i \phi_{n}^{-}}\right)\right] \\
&-(\mu+\kappa)\left[\oint_{\partial \Delta} \arg \frac{1-e^{i \phi_{n}^{+}}}{1-e^{i \phi_{n}^{-}}} d \sigma(\zeta)\right] .
\end{aligned}
$$

The first summand tends to zero while the second one tends to $-\kappa \pi$. The third summand also tends to zero because the integrand is a bounded function which tends to zero for each $\zeta \in \partial \Delta, \zeta \neq 1$. Hence by Lebesgue's bounded convergence theorem, the integral in $(2.32)$ goes to 0.

Then we obtain $2 \beta=-\kappa \pi$, or $\kappa=-2 \beta / \pi<0$. Again by Lemma 2.3 , we get $h_{1} \in \operatorname{Star}_{\mu, \kappa}[1$, $-1]$. The proof is complete.

We now observe by [1] (see also [5]) that each spirallike function $h \in \operatorname{Spiral}[1]=\bigcup_{\mu \in \Omega}$ $\operatorname{Spiral}_{\mu}[1], \Omega=\{\lambda:|\lambda-1| \leq 1, \lambda \neq 0\}$, is a complex power of a starlike function with 
respect to a boundary point. Therefore, one can apply Theorem 2.2 to give geometric characteristics for images of functions of the class Spiral ${ }_{\mu, \nu}[1,-1]$ with complexes $\mu$ and $\nu$. More precisely, let $g_{\lambda, \theta}$ be a univalent function on $\Delta$ defined by

$$
g_{\lambda, \theta}(z)=e^{i \theta \lambda}\left(\frac{1-z}{1+z}\right)^{\lambda}, \quad \lambda \in \Omega, \theta \in \mathbb{R} .
$$

It is clear that $g_{\lambda, \theta} \in \operatorname{Spiral}_{\lambda,-\lambda}[1,-1]$. The set $\widetilde{W_{\lambda, \theta}}:=g_{\lambda, \theta}(\Delta)$ is called a canonical spiral wedge with vertex at the origin.

An immediate consequence of Theorem 2.2 and [1, Theorem 1] is the following assertion.

Theorem 2.4. (i) If $h \in \operatorname{Spiral}_{\mu}[1]$, then the canonical spiral wedge $\widetilde{W_{\lambda, \theta^{*}}}$, where

$$
\theta^{*}:=\lim _{r \rightarrow 1^{-}} \arg h^{1 / \mu}(r),
$$

is the smallest one which contains the image $h(\Delta)$.

(ii) If $h \in \operatorname{Spiral}_{\mu, \nu}[1,-1]$, then the canonical spiral wedge $\widetilde{W_{\lambda, \theta_{*}}}$, where

$$
\theta_{*}:=\lim _{r \rightarrow 1^{-}} \arg h^{1 / \mu}(-r)
$$

is contained in $h(\Delta)$, and there is no canonical spiral wedge $\widetilde{W} \neq \widetilde{W_{\lambda, \theta_{*}}}$ with $\widetilde{W_{\lambda, \theta_{*}}} \subset \widetilde{W} \subset$ $h(\Delta)$.

Conversely, if $h \in$ Spiral $_{\mu}[1]$ and the image $h(\Delta)$ contains some canonical spiral wedge, then there are $\nu(\nu=-r \mu, 0<r \leq 1)$ and an automorphism $\Psi$ of the open unit disk $\Delta$ such that $(1 / h(\Psi(0))) h \circ \Psi \in \operatorname{Spiral}_{\mu, \nu}[1,-1]$.

\section{Functions convex in one direction}

The class of functions we consider here has been studied by several mathematicians (see, e.g., Ciozda [3, 4], Hengartner and Schober [11], Lecko [12]) as a subclass of functions defined by Robertson in [14].

Definition 3.1. Say that a univalent function $h \in \operatorname{Hol}(\Delta, \mathbb{C})$ normalized by

$$
h(0)=0
$$

is convex in the positive direction of the real axis if for each $z \in \Delta$ and $t>0$,

$$
h(z)+t \in h(\Delta), \quad \lim _{t \rightarrow \infty} h^{-1}(h(z)+t)=1 .
$$

The class of those functions is denoted by $\Sigma[1]$.

Here we find the maximal width size of the image for functions of this class using an angular limit characteristic of functions under consideration. In other words, given such a function, we find the minimal horizontal strip which contains its image. The following question is also natural but more complicated: characterize those functions convex in the 
positive direction of the real axis whose images contain a whole (two-sided) strip and find the size (width) of this strip. We solve this problem for functions having maximal horizontal strips of finite size. The problem is still open for the general case.

To proceed, we need the following lemma.

Lemma 3.2 (cf. $[3,4,11]$ ). Let h be a univalent function normalized by (3.1). Then $h \in \Sigma[1]$ if and only if

$$
\operatorname{Re}\left((1-z)^{2} h^{\prime}(z)\right) \geq 0 .
$$

Proof. Let $h \in \Sigma[1]$. By Definition 3.1, for each $t \geq 0$, the holomorphic function $F_{t}$ defined by

$$
F_{t}(z)=h^{-1}(h(z)+t)
$$

maps the unit disk into itself. It is easy to verify that the family $\mathscr{S}=\left\{F_{t}\right\}_{t \geq 0}$ forms a continuous semigroup of holomorphic self-mappings of the unit disk.

Differentiating this semigroup at $t=0^{+}$, we get

$$
f(z):=-\left.\frac{\partial F_{t}(z)}{\partial t}\right|_{t=0^{+}}=-\frac{1}{h^{\prime}(z)}
$$

where $f$ is the so-called infinitesimal generator $f$ of $\mathscr{S}$ (see, e.g., [16]). By (3.2) the point $\tau=1$ is the Denjoy-Wolff point of $\mathscr{S}$ (see, e.g., $[2,16]$ ). Therefore, its generator can be represented by the Berkson-Porta formula (see [2]):

$$
f(z)=-(1-z)^{2} p(z), \quad \text { where } \operatorname{Re} p(z) \geq 0 .
$$

Comparing (3.5) and (3.6) proves inequality (3.3).

Conversely, suppose now that $h$ satisfies (3.3). Then the function $p(z)=1 /(1-z)^{2} h^{\prime}(z)$ has a nonnegative real part.

If $p(z)=i b, b \in \mathbb{R}$, then $h(z)=i b /(1-z)$ belongs to the class $\Sigma[1]$.

If $\operatorname{Re} p(z)>0, z \in \Delta$, then by a result of Berkson and Porta (see [2]), the function $f$ defined by (3.6) is the generator of a semigroup $\mathscr{S}=\left\{F_{t}\right\}_{t \geq 0}$ of holomorphic self-mappings of the unit disk. This semigroup can be defined by the Cauchy problem:

$$
\begin{gathered}
\frac{\partial F_{t}(z)}{\partial t}+f\left(F_{t}(z)\right)=0 \\
F_{0}(z)=z, \quad z \in D .
\end{gathered}
$$

Substituting here $f(z)\left(=-(1-z)^{2} p(z)\right)=-1 / h^{\prime}(z)$, we get

$$
h^{\prime}\left(F_{t}(z)\right) \frac{\partial F_{t}(z)}{\partial t}=1 .
$$

Integrating the latter expression on the interval $[0, t]$, we get

$$
\left.h\left(F_{t}(z)\right)=h(z)+t \quad \text { (i.e., } h(z)+t \in h(\Delta)\right) .
$$


Since $\mathscr{Y}$ has a Denjoy-Wolff point at 1 , it follows that

$$
\lim _{t \rightarrow \infty} h^{-1}(h(z)+t)\left(=\lim _{t \rightarrow \infty} F_{t}(z)\right)=1 .
$$

This completes our proof.

Lemma 3.3. Let $h \in \Sigma[1]$. Then the limit

$$
\angle \lim _{z \rightarrow 1}(1-z) h^{\prime}(z)=\mu
$$

is either a positive real number or infinity.

Proof. By Lemma 3.2, the function

$$
p(z)=\frac{1}{(1-z)^{2} h^{\prime}(z)}
$$

is either of positive real part or an imaginary constant. In the latter case, $p(z)=i b, b \in \mathbb{R}$, and the assertion is evident. Otherwise, one can write

$$
\frac{1}{(1-z) h^{\prime}(z)}=(1-z) p(z)
$$

It was proved in [8] (see also [17]) that for any function $p$ with positive real part, the angular limit $\angle \lim _{z \rightarrow 1}(1-z) p(z)$ exists and is a nonnegative real number. This proves our assertion.

Definition 3.4. Say that a univalent function $h$ belongs to the class $\Sigma_{\mu}[1]$ with $\mu, 0<\mu \leq$ $\infty$, if it is of the class $\Sigma[1]$ and the limit (3.11) is equal to $\mu$.

Thus by Lemma 3.3, we have $\Sigma[1]=\bigcup_{0<\mu \leq \infty} \Sigma_{\mu}[1]$.

Example 3.5. The function

$$
h_{1}(z)=\log \frac{1+z}{1-z}
$$

belongs to $\Sigma_{1}[1]$, while the function

$$
h_{2}(z)=\frac{z}{1-z}
$$

belongs to $\Sigma_{\infty}[1]$.

Definition 3.6. Say that a univalent function $h$ belongs to the class $\Sigma_{\mu, \nu}[1,-1]$ if it is of the class $\Sigma_{\mu}[1]$ and satisfies the conditions

$$
\begin{aligned}
& \angle \lim _{z \rightarrow-1} \operatorname{Re} h(z)=-\infty \\
& \angle \lim _{z \rightarrow-1}(z+1) h^{\prime}(z)=v .
\end{aligned}
$$


Theorem 3.7. (i) If $h \in \Sigma_{\mu}[1], \mu \in(0, \infty)$, then the horizontal strip

$$
S^{*}=\left\{z:\left|\operatorname{Im} z-a^{*}\right|<\frac{\pi \mu}{2}\right\} \quad \text { with } a^{*}=\lim _{r \rightarrow 1^{-}} \operatorname{Im} h(r)
$$

is the smallest one which contains the image $h(\Delta)$. If $h \in \Sigma_{\infty}[1]$, then there is no horizontal strip of finite width containing $h(\Delta)$.

(ii) If $h \in \Sigma_{\mu, \nu}[1,-1], \mu \in(0, \infty)$, then the horizontal strip

$$
S_{*}=\left\{z:\left|\operatorname{Im} z-a_{*}\right|<\frac{\pi \nu}{2}\right\} \quad \text { with } a_{*}=\lim _{r \rightarrow 1^{-}} \operatorname{Im} h(-r)
$$

is contained in $h(\Delta)$, and there is no strip $S \neq S_{*}$ with $S_{*} \subset S \subset h(\Delta)$. Consequently, $v \in$ $(0, \mu]$.

Conversely, if $h \in \Sigma_{\mu}[1], \mu \in(0, \infty)$, and the image $h(\Delta)$ contains some open horizontal strip, then there are $\nu(0<\nu \leq \mu)$ and an automorphism $\Psi$ of the open unit disk $\Delta$ such that $h(\Psi(\cdot))-h(\Psi(0))$ belongs to $\Sigma_{\mu, \nu}[1,-1]$ (see Figure 3.1).

Proof. (i) Suppose that $h \in \Sigma_{\mu}[1], \mu \in(0, \infty)$, that is, the limit (3.11) is finite. By Lemma 3.2 , the function $p(z)=(1-z)^{2} h^{\prime}(z)$ is of nonnegative real part. Hence, there exists a self-mapping $\omega(z)$ such that

$$
(1-z)^{2} h^{\prime}(z)=4 \mu \frac{1-\omega(z)}{1+\omega(z)}
$$

Thus

$$
\frac{1-\omega(z)}{1-z}=\frac{1+\omega(z)}{4} \frac{(1-z) h^{\prime}(z)}{\mu} .
$$

Since the right-hand side of this equality is bounded on each nontangential approach region at the point $z=1$, one concludes that $\angle \lim _{z \rightarrow 1} \omega(z)=1$. Now we calculate

$$
\angle \lim _{z \rightarrow 1} \frac{1-\omega(z)}{1-z}=\angle \lim _{z \rightarrow 1} \frac{1+\omega(z)}{4} \frac{(1-z) h^{\prime}(z)}{\mu}=\frac{1}{2} \in(0,1) .
$$

Consider the function

$$
g(z)=\exp \left[-\frac{1}{\mu} h(z)\right]
$$

It satisfies

$$
-\frac{g^{\prime}(z)}{g(z)}(1-z)^{2}=4 \frac{1-\omega(z)}{1+\omega(z)}
$$

So, by a result of Lecko and Lyzzaik [13], the function $g$ is univalent and starlike with respect to a boundary point. Since

$$
\angle \lim _{z \rightarrow 1} \frac{(z-1) g^{\prime}(z)}{g(z)}=1,
$$




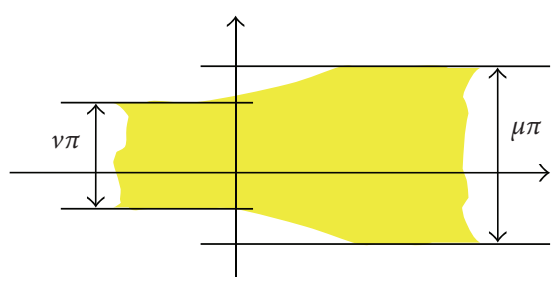

Figure 3.1. Width distortion.

we see that $g \in \operatorname{Star}_{\lambda}[1]$ with $\lambda=1$. By Theorem 2.2, the smallest wedge which contains the image $g(\Delta)$ is defined by $(2.2)$ :

$$
W^{*}=\left\{w \in \mathbb{C}:\left|\arg w-\theta_{+}\right|<\frac{\pi}{2}\right\},
$$

where

$$
\theta^{*}=\lim _{r \rightarrow 1^{-}} \arg g(r)=\lim _{r \rightarrow 1^{-}}-\frac{\operatorname{Im} h(r)}{\mu} .
$$

Therefore the smallest horizontal strip which contains the image of function $h(z)=$ $-\mu \log g(z)$ is defined by (3.17).

Suppose now that $h \in \Sigma[1]$ and its image is contained in a horizontal strip of finite width $\pi \mu(0<\mu<\infty)$. In this case, the function $g$ defined by (3.22) is univalent and starlike with respect to a boundary point. Moreover, since the minimal strip which contains the image $h(\Delta)$ is of width $\pi \mu$, the minimal wedge containing $g(\Delta)$ is of angle $\pi$. Once again by Theorem 2.2, $g \in \operatorname{Star}_{\lambda}[1]$ with $\lambda=1$; hence it satisfies the Visser-Ostrowski condition

$$
\angle \lim _{z \rightarrow 1} \frac{(z-1) g^{\prime}(z)}{g(z)}=1
$$

Since

$$
\frac{(z-1) g^{\prime}(z)}{g(z)}=\frac{(1-z) h^{\prime}(z)}{\mu}
$$

and $0<\mu<\infty$, we have that $h \in \Sigma_{\mu}[1]$.

(ii) As above, define a starlike function $g$ by (3.22). It is clear that $\lim _{r \rightarrow 1^{-}} g(-r)=\infty$. Since $g$ is univalent, we conclude that $\angle \lim _{z \rightarrow-1} g(z)=\infty$. Furthermore,

$$
\angle \lim _{z \rightarrow-1} \frac{(z+1) g^{\prime}(z)}{g(z)}=\angle \lim _{z \rightarrow-1} \frac{-(z+1) h^{\prime}(z)}{\mu}=-\frac{\nu}{\mu} .
$$

So, $g \in \operatorname{Star}_{1,-v / \mu}[1,-1]$. As we mentioned above, in this case $-1 \leq-v / \mu \leq 0$, that is, $\nu \in[0, \mu]$. By Theorem 2.2, the wedge

$$
W_{*}=\left\{w:\left|\arg w-\theta_{*}\right|<\frac{\nu \pi}{2 \mu}\right\} \quad \text { with } \theta_{*}=\lim _{r \rightarrow 1^{-}} \arg g(-r)
$$


is contained in $g(\Delta)$, and there is no wedge $W \neq W_{*}$ with $W_{*} \subset W \subset g(\Delta)$. Since $h(z)=$ $-\mu \log g(z)$, we have that the limit $a_{*}=\lim _{x \rightarrow-1^{+}} \operatorname{Im} h(x)=-\mu \theta_{*}$ exists. In addition, the strip $S_{*}$ defined by (3.18) is contained in $h(\Delta)$, and there is no strip $S \neq S_{*}$ with $S_{*} \subset S \subset$ $h(\Delta)$.

Conversely, suppose that $h \in \Sigma_{\mu}[1]$ and its image $h(\Delta)$ contains an open strip. Then the function $g$ defined by (3.22) belongs to $\operatorname{Star}_{\mu}[1]$ and its image $g(\Delta)$ contains an open wedge. By Theorem 2.2, we conclude that there are a negative number $\nu$ and an automorphism $\Psi$ such that the function $g_{1}:=(1 / g(\Psi(0))) g \circ \Psi$ belongs to $\operatorname{Star}_{\mu, \nu}[1,-1]$. In this case,

$$
h(\Psi(z))-h(\Psi(0))=-\mu \log g_{1}(z) \in \Sigma_{\mu, \nu}[1,-1] .
$$

This completes our proof.

Remark 3.8. Unfortunately, the problem of finding a maximal strip which is contained in the image $h(\Delta)$ is still open when $h \in \Sigma_{\infty}[1]$.

This problem is a key to solve the following important problem in the theory of semigroups of parabolic type.

Given a semigroup $\mathscr{S}=\left\{F_{t}\right\}_{t \leqslant 0}$ of holomorphic self-mappings of $\Delta$, find an open subset $\Omega \in \Delta$ such that $\mathscr{S} \subset \operatorname{Aut}(\Omega)$.

If $\mathscr{S}=\left\{F_{t}\right\}_{t \leqslant 0}$ has a Denjoy-Wolff point $\tau \in \partial \Delta$ of parabolic type, that is, $F_{t}^{\prime}(\tau)=1$ for all $t \geq 0$, then there is a solution of Abel's functional equation

$$
h\left(F_{t}(z)\right)=h(z)+t
$$

(see, e.g., [10]) which is of the class $\Sigma_{\infty}$ [1]. If $\mathscr{Y}=\left\{F_{t}\right\}_{t \leqslant 0}$ has a repelling boundary fixed point, then this problem is equivalent to the existence of an open strip which is contained in $h(\Delta)$.

\section{Acknowledgment}

We are very grateful to the referees for useful remarks and comments.

\section{References}

[1] D. Aharonov, M. Elin, and D. Shoikhet, Spiral-like functions with respect to a boundary point, Journal of Mathematical Analysis and Applications 280 (2003), no. 1, 17-29.

[2] E. Berkson and H. Porta, Semigroups of analytic functions and composition operators, Michigan Mathematical Journal 25 (1978), no. 1, 101-115.

[3] K. Ciozda, Sur la classe des fonctions convexes vers l'axe réel négatif, Bulletin de l'Académie Polonaise des Sciences. Série des Sciences Mathématiques 27 (1979), no. 3-4, 255-261.

[4] __ Sur quelques problèmes extrémaux dans les classes des fonctions convexes vers l'axe réel négatif, Annales Polonici Mathematici 38 (1980), no. 3, 311-317.

[5] M. Elin, A. Goldvard, S. Reich, and D. Shoikhet, Dynamics of spirallike functions, Complex Analysis and Dynamical Systems, Contemp. Math., vol. 364, American Mathematical Society, Rhode Island, 2004, pp. 41-57.

[6] M. Elin, S. Reich, and D. Shoikhet, Holomorphically accretive mappings and spiral-shaped functions of proper contractions, Nonlinear Analysis Forum 5 (2000), 149-161. 
[7] __ Dynamics of inequalities in geometric function theory, Journal of Inequalities and Applications 6 (2001), no. 6, 651-664.

[8] M. Elin and D. Shoikhet, Dynamic extension of the Julia-Wolff-Carathéodory theorem, Dynamic Systems and Applications 10 (2001), no. 3, 421-437.

[9] Univalent functions of proper contractions spirallike with respect to a boundary point, Multidimensional Complex Analysis, Krasnoyarsk, 2002, pp. 28-36.

[10] T. E. Harris, The Theory of Branching Processes, Die Grundlehren der mathematischen Wissenschaften, vol. 119, Springer, Berlin, 1963.

[11] W. Hengartner and G. Schober, On Schlicht mappings to domains convex in one direction, Commentarii Mathematici Helvetici 45 (1970), 303-314.

[12] A. Lecko, On the class of functions convex in the negative direction of the imaginary axis, Journal of the Australian Mathematical Society 73 (2002), no. 1, 1-10.

[13] A. Lecko and A. Lyzzaik, A note on univalent functions starlike with respect to a boundary point, Journal of Mathematical Analysis and Applications 282 (2003), no. 2, 846-851.

[14] M. S. Robertson, Analytic functions star-like in one direction, American Journal of Mathematics 58 (1936), no. 3, 465-472.

[15] Univalent functions starlike with respect to a boundary point, Journal of Mathematical Analysis and Applications 81 (1981), no. 2, 327-345.

[16] D. Shoikhet, Semigroups in Geometrical Function Theory, Kluwer Academic, Dordrecht, 2001.

[17] _ Representations of holomorphic generators and distortion theorems for spirallike functions with respect to a boundary point, International Journal of Pure and Applied Mathematics 5 (2003), no. 3, 335-361.

Mark Elin: Department of Mathematics, ORT Braude College, P.O. Box 78, Karmiel 21982, Israel E-mail address: mark.elin@gmail.com

David Shoikhet: Department of Mathematics, ORT Braude College, P.O. Box 78, Karmiel 21982, Israel

E-mail addresses: davs27@netvision.net.il; brau_math@admin.ort.org.il 


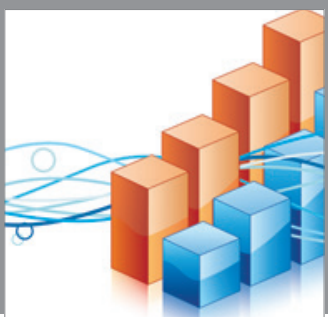

Advances in

Operations Research

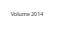

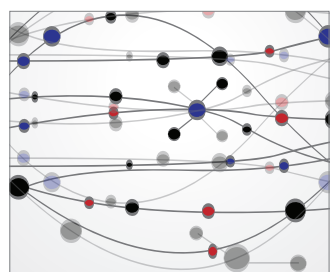

\section{The Scientific} World Journal
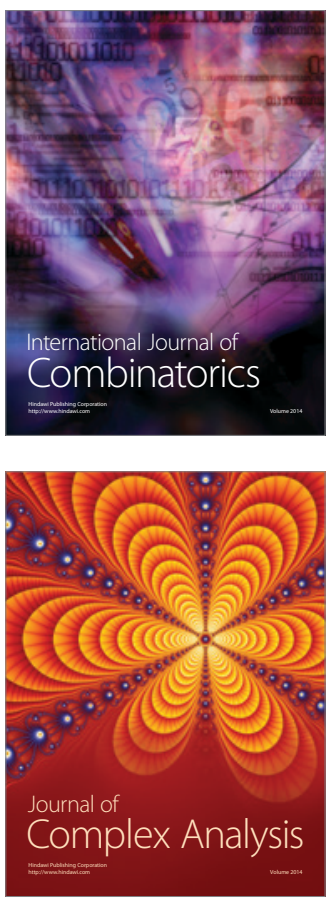

International Journal of

Mathematics and

Mathematical

Sciences
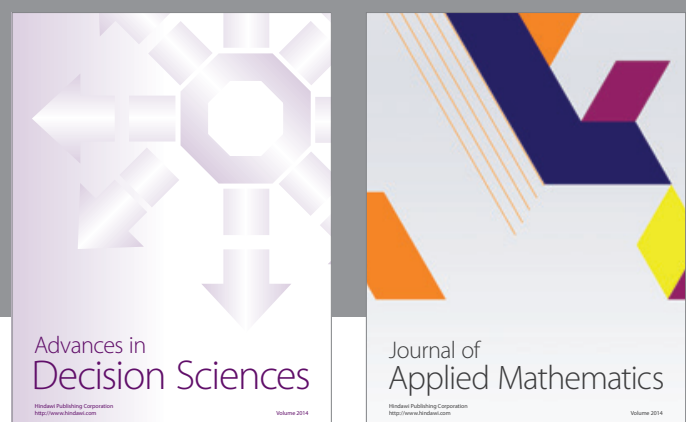

Journal of

Applied Mathematics
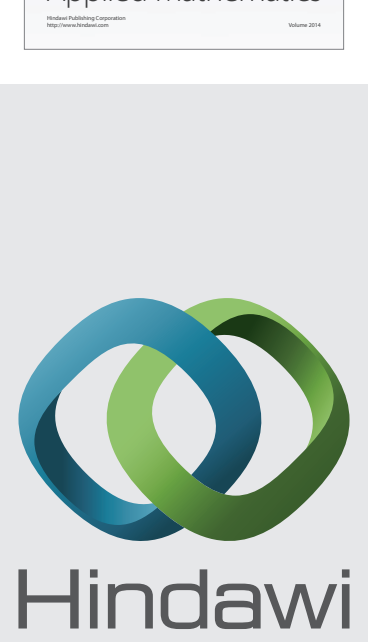

Submit your manuscripts at http://www.hindawi.com
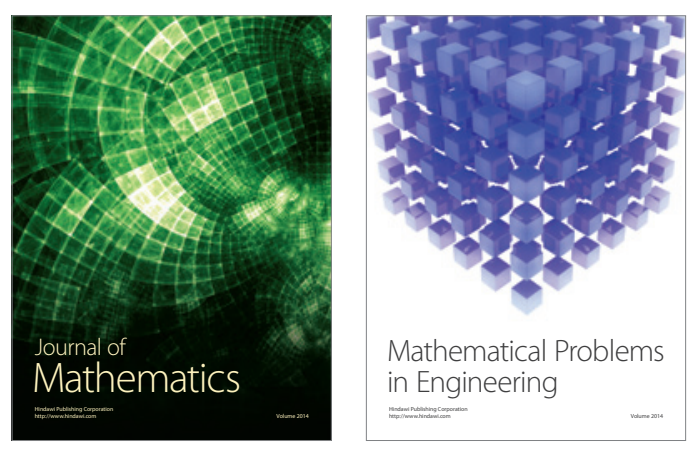

Mathematical Problems in Engineering
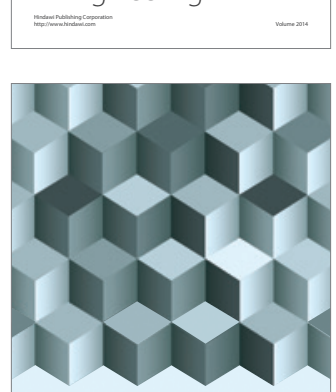

Journal of

Function Spaces
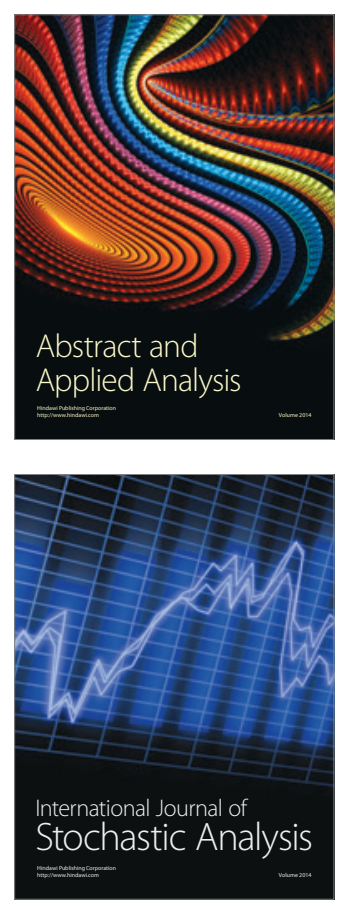

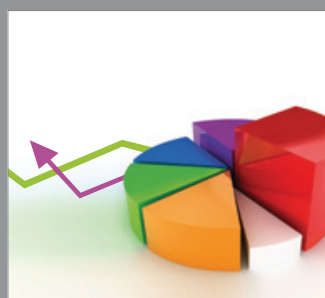

ournal of

Probability and Statistics

Promensencen
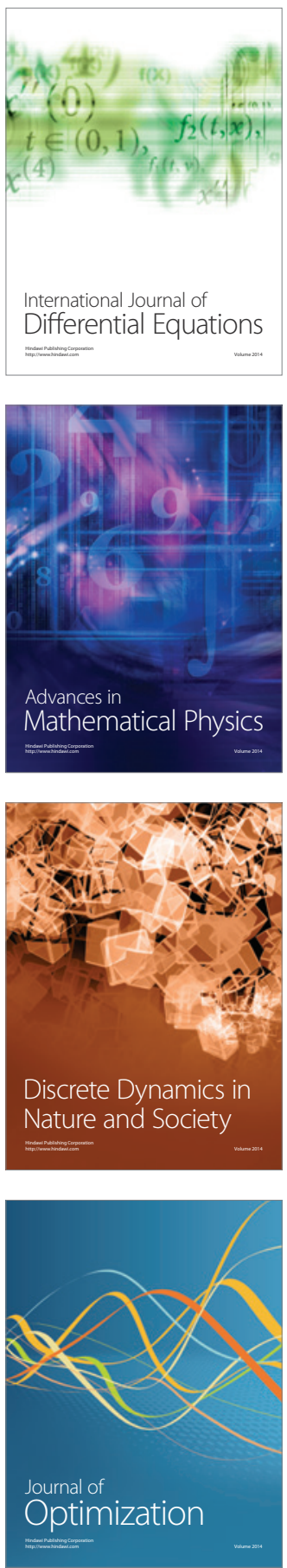